\title{
La performance: Una propuesta interdisciplinar de las áreas de educación física, educación musical y educación visual y plástica en la formación inicial de los futuros maestros The performance: an interdisciplinary proposal from the departments of physical education, music education and visual arts education in the initial teacher training
}

Carmina Folch Dávila, Txema Córdoba Jiménez, Dolors Ribalta Alcalde

Universidad Ramón Llull (España)

\begin{abstract}
Resumen. En este artículo presentamos una propuesta pedagógica interdisciplinar que se inscribe en el módulo Educación a través de la plástica, la música y la educación física: proyecto interdisciplinar de los grados de Educación Infantil y Primaria de la Universidad Ramon Llull. La actividad nuclear de este módulo es una performance: una acción artística como resultado de un proceso creativo interdisciplinar, tutorizado por tres docentes de las áreas de educación física, educación musical y educación visual y plástica. Los objetivos del artículo consisten en describir este proceso creativo, incidiendo en las fases del aprendizaje interdisciplinar, la codocencia, el aprendizaje cooperativo y la evaluación formativa, y reflexionar sobre los resultados obtenidos y las condiciones para su implementación. Entre las conclusiones, destacamos la contribución del proyecto interdisciplinar a la formación personal y profesional de los futuros maestros y maestras, el reto que supone para los estudiantes la integración interdisciplinar en un proceso creativo y la importancia de la codocencia en un equipo multidisciplinar para favorecer la síntesis entre contenidos y lenguajes expresivos. Finalmente, se demuestra que el proyecto reúne todas las condiciones del aprendizaje cooperativo que se ven fortalecidas por la inclusión de un sistema de evaluación formativa basada en rúbricas consensuadas con el alumnado y tutorías.
\end{abstract}

Palabras clave: interdisciplinariedad, proceso creativo, codocencia, aprendizaje cooperativo, evaluación formativa.

Abstract. In this article we present an interdisciplinary pedagogical proposal that is part of the module Education through visual arts, music and physical education: interdisciplinary project of the Early Childhood and Primary Education degrees of Ramon Llull University. The nuclear activity of this module is a performance: an artistic action, a result of an interdisciplinary creative process, supervised by three teachers from the departments of physical education, music education, and visual arts education. The objectives of the article are to describe this creative process, focusing on the phases of interdisciplinary learning, co-teaching, cooperative learning, and formative evaluation; and to reflect on the results obtained and the conditions for its implementation. Among the conclusions, we highlight the contribution of the interdisciplinary project to the personal and professional training of future teachers, the interdisciplinary integration in a creative process as a challenge for students, and the importance of co-teaching in a multidisciplinary team to promote synthesis between contents and expressive languages. Finally, it is shown that the project meets all the conditions of cooperative learning, strengthened by the inclusion of a formative evaluation system based on agreed-upon rubrics with the students and tutorials.

Key words: interdisciplinarity, creative process, coteaching, cooperative learning, formative assessment.

\section{Introducción}

La revolución digital del siglo XXI ha generado nuevas necesidades de formación y ha comportado la eclosión de múltiples iniciativas de renovación pedagógica en las escuelas e instituciones de educación formal. Estas iniciativas requieren un mayor compromiso del profesorado en las comunidades educativas y su entorno y una ampliación de sus competencias docentes (Cela \& Domènech, 2016; Civís, 2017; Fontanet, 2017; Imbernón \& Colén, 2015; Marina, Pellicer \& Manso, 2015). En este contexto de cambio permanente y acelerado, la formación inicial de los futuros maestros y maestras debe responder al nuevo perfil que demandan las escuelas, con propuestas innovadoras y coherentes con el carácter competencial de los actuales grados de educación infantil y primaria.

En esta encrucijada, planteamos una propuesta práctica desarrollada en el módulo Educación a través de la plástica, la música y la educación física: proyecto interdisciplinar que se imparte en el tercer curso de los Grados de Educación Infantil y Educación Primaria de la Facultat de Psicologia, Ciències de l’Educació i de l’Esport Blanquerna.

\footnotetext{
Fecha recepción: 12-02-19. Fecha de aceptación: 08-09-19

Txema Córdoba Jiménez
}

josemariacj@blanquerna.url.edu
Se trata de una asignatura basada en la puesta en práctica de un proceso creativo a partir de la expresión artística (corporal, musical, visual y plástica). Esta materia pretende, por un lado, promover el desarrollo personal del alumnado potenciando sus capacidades expresivas y, por otro, contribuir a su desarrollo social, despertando su sensibilidad hacia las manifestaciones artísticas y culturales de su entorno. Según López-Pastor, Pérez-Brunicardi, Manrique y Monjas (2016) el principal reto de la educación física actual consiste en clarificar su finalidad y su papel en la educación formal. En el proyecto que presentamos, las áreas de educación física, música y educación visual y plástica comparten la finalidad de contribuir al desarrollo integral del alumnado a través de una propuesta interdisciplinar.

A lo largo de este artículo nos proponemos profundizar en la implementación de esta propuesta didáctica y en sus implicaciones en la formación inicial del profesorado. En este sentido, nos planteamos los siguientes objetivos: (a) describir una propuesta pedagógica en la formación inicial de maestros que incide en la interdisciplinariedad, el proceso creativo, la codocencia, el aprendizaje cooperativo y la evaluación formativa y (b) establecer reflexiones y consideraciones para la puesta en práctica de proyectos artísticos en la formación inicial de los futuros maestros. 


\section{Estado de la cuestión}

Aprendizaje interdisciplinar y proceso creativo como formas de comprensión de la realidad

El Espacio Europeo de Educación Superior (EEES) y los resultados de las investigaciones en educación describen un perfil docente que debería traspasar los límites del aula, capaz de trabajar en equipo, de cooperar en la escuela, de implicarse en su entorno, comprometido con la educación de sus alumnos y con vocación de transformación social (Cela \& Domènech, 2016; Civís, 2017; Fontanet, 2017; Imbernón \& Colén, 2015; Marina, et al., 2015). Sin embargo, los actuales planes de estudio de los grados de educación, fragmentados en asignaturas, siguen formando un perfil de docente centrado en el aula (Imbernón \& Colén, 2015).

Para dar respuesta a las necesidades de la escuela de hoy, la formación inicial de los futuros maestros y maestras debería ser interdisciplinar y coherente con el carácter competencial de los actuales planes de estudio de los grados de educación, además de incluir la investigación y permitir a los estudiantes experimentar las metodologías que aplicarán en su futuro como docentes (EHEA, 2018; Fontanet, 2017; Martínez \& Prats, 2018; UNESCO, 2015). En este sentido, la interdisciplinariedad en la formación universitaria permite una mayor conexión de los currículums con la realidad y la práctica (Cela \& Domènech, 2016). Además, el currículum integrado aporta beneficios a la formación de los estudiantes porque ayuda a desarrollar capacidades cognitivas complejas y facilita la transferencia de conocimientos. A su vez, evita repeticiones en los planes de estudio y aumenta la motivación de estudiantes y profesorado (Bautista, Tan, Ponnusamy \& Yau, 2015; Follari, 2007, 2013).

El objetivo del aprendizaje interdisciplinar es el progreso en la comprensión de la realidad que nos proponemos estudiar, a través de múltiples sistemas simbólicos y distintas formas de representación, incluidas las manifestaciones artísticas (Boix, 2016). Esta forma de aprendizaje se construye en cuatro fases en continua interacción, cada una de las cuales requiere la orientación del profesorado (Boix 2016):

- La definición del propósito de investigación.

- La investigación disciplinar en la cual se valoran y comparan las aportaciones de cada disciplina a la resolución del problema.

- La síntesis interdisciplinar.

- El análisis crítico de los resultados en relación al propósito previamente definido.

Este proceso es la base de las distintas formas de integración interdisciplinar, desde los estudios científicos hasta las producciones artísticas y tiene mucho en común con el proceso creativo que describen Batet, Ponti \& Segarra (2015). Para estos autores, creatividad significa establecer conexiones entre distintas realidades y buscar nexos de unión donde aparentemente no hay nada en común, investigando más allá de lo que nos es conocido. Así, el proceso creativo se desarrolla en cuatro etapas (Batet et al. 2015):

- Inmersión: conocer, buscar información, adquirir un marco referencial.

- Reflexión: ordenar los datos y las sensaciones que se han experimentado durante la inmersión.

- Ideación: generar ideas para crear el mayor número de alternativas creativas posibles, buscando la originalidad.

- Revisión: validar las propuestas y reconducirlas si fuera necesario.

El proceso creativo, tal y como lo describen Batet et al. (2015) tiene mucho en común con el modelo de aprendizaje interdisciplinar desarrollado por Boix (2016), puesto que esta autora considera que las manifestaciones artísticas son el resultado de un proceso de aprendizaje multidisciplinar en el cual los estudiantes deben integrar la información que aportan las distintas disciplinas sobre el objeto de estudio y sobre las múltiples formas de representarlo. Para ello es imprescindible la orientación del profesorado, porque producir síntesis reveladoras y estéticas requiere conocimiento sobre cómo se crean metáforas. Según Acaso (2015), el arte contemporáneo debería ser el principal recurso para introducir lo metafórico en la educación formal, porque suele ser multilenguaje, narrativo y trata temas vinculados a la realidad social, por lo que conecta con los intereses de los estudiantes.

A pesar de los beneficios que aporta, la aplicación del aprendizaje interdisciplinar es todavía poco frecuente en las distintas etapas del sistema educativo, incluida la universidad, a causa de las dificultades que el profesorado debe afrontar: rigidez de currículums, evaluación estandarizada y falta de espacios de colaboración entre profesores de distintas áreas (Bautista, et al., 2015; Follari, 2013). Según Follari (2013), ningún profesor puede ser interdisciplinario por sí solo ya que «descentrarse de un punto de vista singular es decisivo en ese proceso; y ello supone al grupo y se da sólo como efecto del mismo» (p. 127).

Cuando un grupo de profesores implicado en un proceso interdisciplinar comparte aula, hablamos de codocencia. Ésta se define como la actuación de dos o más docentes comprometidos en un proceso de enseñanza colaborativa, destinada a un único grupo de estudiantes, en un mismo espacio y tiempo, con la finalidad de promover el aprendizaje de determinados contenidos que no hubieran podido enseñar trabajando de manera individual (Cook, 2004; SuárezDíaz, 2016). Entre los distintos modelos de codocencia, el denominado «equipo docente» es el que genera más interacción entre docentes y entre docentes y estudiantes, porque los profesores implicados desempeñan la misma función de forma simultánea sin que destaque entre ellos un liderazgo visible (Cook, 2004; Suárez-Díaz, 2016).

Diferentes autores defienden que la figura del profesor único pertenece a un modelo de docencia del pasado (Acaso, 2015; MacBeath, 2013; Schleicher, 2015). Para Shleicher (2015), las escuelas más eficientes agrupan docentes que comparten un mismo espacio, tiempo y alumnado, agrupan estudiantes de distintas edades y capacidades y unen franjas horarias para permitir procesos de aprendizaje largos y profundos. La presencia de más de un docente en los espacios educativos favorece el paso de la educación industrial a la educación artesanal centrada en el alumno (Acaso, 2015), a la vez que contribuye al crecimiento profesional del profesorado, que adquiere nuevas estrategias, aprende cómo aplicarlas e incrementa su satisfacción personal y autoestima (Acaso, 2015; MacBeath, 2013; Schleicher, 2015; Suárez-Díaz, 2016).

La codocencia es un reto importante porque obliga a las 
instituciones educativas a cuestionarse muchos esquemas adquiridos durante décadas: el profesor solo frente a un único grupo de alumnos, los grupos separados unos de otros, los horarios y asignaturas compartimentados y las formas tradicionales de organización del aula (Schleicher, 2015; SuárezDíaz, 2016). En el contexto universitario, los resultados obtenidos por Suárez-Díaz (2016) muestran que la mayoría del profesorado entiende la codocencia como un proceso de formación totalmente compartido, pero muy pocos saben cómo debe ser el diseño didáctico y de la evaluación que permita una visión integrada y evite la fragmentación en asignaturas. En la formación inicial de maestros, la codocencia constituye un modelo de práctica colaborativa para los futuros docentes (Folch, Capdevila \& Prats, 2019; Kluth \& Straut, 2003).

\section{Aprendizaje cooperativo y evaluación formativa, dos procesos indisociables}

El aprendizaje cooperativo es el planteamiento educativo basado en la interacción entre personas a partir de propuestas, estructuras y actividades que aseguran una interdependencia positiva entre los participantes, quienes persiguen un objetivo común (Johnson, Johnson \& Holubec, 1999; Velázquez-Callado, 2010). La importancia de su utilización se contempla desde dos perspectivas; por un lado, por los beneficios que aporta a nivel de resultados académicos cualitativos y cuantitativos (Johnson et al., 1999) y, por otro, por el desarrollo de valores propios de la cooperación, tan necesarios en el contexto social actual (Pujolàs, 2008). Se está convirtiendo en una metodología básica dentro del modelo de educación basado en competencias y se puede aplicar al aprendizaje de cualquier contenido (Johnson \& Johnson, 2014; Pujolàs, 2008; Velázquez-Callado, 2015).

Para provocar que la metodología de aprendizaje sea cooperativa no basta con agrupar a los alumnos en equipos de trabajo. Es necesario que el docente diseñe tareas donde se cumplan cinco condiciones básicas (Johnson et al., 1999):

- Interdependencia positiva: el alumnado debe apreciar que el éxito individual de cada uno está vinculado al éxito de los demás.

- Interacción promotora cara a cara: asistencia mutua, feedback permanente y actitudes motivadoras entre los miembros del grupo.

- Responsabilidad y valoración personal: rendición de cuentas personal y evaluación del avance individual.

- Habilidades interpersonales: el alumnado debe desarrollar habilidades para una gestión del grupo eficaz, como la confianza, el apoyo mutuo, la resolución de conflictos y la comunicación precisa y sincera.

- Procesamiento grupal: reflexión acerca del funcionamiento del grupo, su eficacia y los resultados obtenidos con el fin de realizar cambios y mejoras para el futuro.

Numerosos estudios defienden la necesidad de lograr una fuerte coherencia entre las metodologías cooperativas y los sistemas de evaluación formativa y compartida, especialmente aquellos que se centran en la autorregulación del aprendizaje por parte del alumnado (Córdoba, López-Pastor \& Sebastiani, 2018; Fernández-Río, 2017; Johnson \& Johnson, 2014; Pujolás, 2008; Velázquez-Callado, 2010).

En este sentido, López-Pastor (2009) define la evalua- ción formativa como el proceso de evaluación cuya finalidad principal es mejorar los procesos de enseñanza-aprendizaje incidiendo en tres aspectos clave: el proceso de aprendizaje del alumnado; la práctica profesional del docente y las actividades que se desarrollan en el aula. Se trata de un sistema de evaluación conceptualmente opuesto al sistema tradicional, llamado evaluación sumativa o de evaluación-calificación (López-Pastor, 2006; López-Pastor \& Pérez-Pueyo, 2017), que orienta sus prácticas a obtener una calificación mediante la realización de un examen o trabajo final, o bien a través de varios exámenes o trabajos parciales. La evaluación sumativa entiende al alumno como actor pasivo del proceso educativo y se centra de forma exclusiva y obsesiva en la calificación. Suprime el deseo de aprender y relega el proceso educativo al hecho de ir superando obstáculos y niveles propuestos por el profesor, en base a un intercambio de trabajos y tiempo de estudio por notas (Álvarez-Méndez, 2001; Santos-Guerra, 2015).

En el campo de la educación física, el concepto tradicional de evaluación se ha basado en un modelo conductista orientado a conseguir objetivos de rendimiento mesurables para la calificación y selección del alumnado (Velázquez \& Hernández, 2005).

En cambio, la evaluación formativa no busca calificar al alumnado, sino que consiste en un proceso sin carácter sancionador, que se desarrolla de manera sistemática a lo largo de todo el proceso educativo. Tiene como finalidad obtener información que sirva tanto para ayudar al alumnado a mejorar, haciéndolo consciente de su aprendizaje, como para que los docentes realicen su trabajo cada vez mejor, reflexionando sobre su práctica educativa (López-Pastor \& Pérez-Pueyo, 2017). Desde esta perspectiva, teniendo en cuenta al alumnado como parte implicada en el hecho educativo, éste puede participar en el proceso de evaluación de distintas formas (López-Pastor, 2009): en la autoevaluación, cuando se evalúa a sí mismo; en la coevaluación o evaluación entre iguales, cuando evalúa a sus compañeros y en la evaluación compartida, cuando dialoga con el profesorado respecto al proceso de aprendizaje que está teniendo lugar.

Una evaluación formativa y compartida coherente con las metodologías cooperativas de aprendizaje debería poseer las siguientes características:

- Estar integrada en el proceso de aprendizaje grupal y cooperativo.

- Desarrollar procesos de autoevaluación y coevaluación.

- Utilizar instrumentos de evaluación variados.

- Valorar tanto los logros motores como los cognitivos, sociales y actitudinales.

La performance. Un ejemplo de aprendizaje interdisciplinar a través de los lenguajes corporal, musical, visual y plástico

En el módulo Educación a través de la plástica, la música y la educación física: proyecto interdisciplinar participan conjuntamente tres docentes de las áreas de educación física, educación musical y educación visual y plástica. Los resultados de aprendizaje que se persiguen son los siguientes: 
1. Conocer la especificidad de los lenguajes propios de cada disciplina (sonoro, visual, corporal) así como la transposición a otros lenguajes.

2. Adquirir competencias artísticas a partir de la práctica y de la investigación mostrando rigor en las actividades propuestas.

3. Desarrollar un proceso creativo interdisciplinar a través del trabajo en equipo, ejerciendo liderazgo y con una actitud participativa durante todo el proceso.

4. Proyectar el trabajo artístico interdisciplinar en un entorno escolar a partir de una propuesta educativa mostrando una visión innovadora, abierta y creativa.

El proyecto se divide en dos partes que corresponden a cada uno de los dos semestres del curso. En la primera parte se realizan actividades relacionadas con un tema propuesto por el profesorado, cuyo objetivo es ofrecer a los estudiantes recursos expresivos, iniciarlos en el proceso creativo y, a la vez, ayudarlos a adquirir confianza en sus propias capacidades comunicativas.

Durante esta primera fase del curso desde el área de educación física incidimos en el movimiento como un medio para la expresión, la comunicación y el desarrollo de la creatividad y por ello nuestras propuestas se basan en la experimentación y el diálogo que permite a los alumnos decidir y realizar el gesto, el movimiento o la coreografía que mejor responde a sus objetivos. A modo de ejemplo, algunas de las actividades que hemos realizado en los últimos cursos han consistido en:

- Representar un personaje de un texto a través de una breve secuencia de movimientos, una composición sonora y una imagen, siendo los alumnos quienes crean la secuencia, la composición y la imagen.

- Crear un poema visual a partir de una idea, con materiales previamente escogidos por el profesorado y recitar el poema utilizando la voz y el gesto como recursos expresivos.

En este artículo nos centramos en la performance, la actividad nuclear del segundo semestre. Esta actividad se basa en un proceso creativo y de investigación que se desarrolla en grupos de 5 o 6 estudiantes y termina con una producción artística en formato de "performance», es decir, una acción artística en vivo. Ésta, es el resultado de un proceso de aprendizaje interdisciplinar, que consta de cuatro fases en continua interacción (Boix, 2016): la definición del propósito, la investigación disciplinar, la síntesis interdisciplinar y el análisis crítico del resultado. En cada una de las sesiones, el alumnado trabaja en equipo de manera autónoma mientras el profesorado se va reuniendo con cada uno de los grupos para ofrecer feedback, lo más inmediato posible, sobre el proceso que están llevando a cabo.

En la fase de definición del propósito, cada grupo de estudiantes escoge un tema de interés común sobre el que investigará para descubrir todas sus posibilidades de desarrollo. Es frecuente que los alumnos elijan temas vinculados a algunas asignaturas que han cursado (como la psicología o la sociología) y temas vinculados a la actualidad que les preocupan. Algunos ejemplos de temas recurrentes son el desarrollo de la identidad, las emociones, el cuerpo, el feminismo, la situación de los refugiados o la sostenibilidad medioambiental.

Una vez han escogido la temática, desde las primeras conversaciones, los estudiantes describen e ilustran su proceso creativo en un diario donde recogen los temas propuestos, los acuerdos y desacuerdos del grupo y las dudas iniciales. En fases posteriores, en este mismo diario, también irán incluyendo los resultados de la investigación, las propuestas de integración interdisciplinar y sus posibles formas de expresión, así como los ensayos y los detalles sobre los materiales y condiciones necesarias para su presentación final.

En la fase de investigación disciplinar, las tutorías compartidas por los tres docentes cobran especial importancia. Desde su formación disciplinar, el equipo de profesores sugiere referentes teóricos y artísticos que cada grupo incorpora a su investigación sobre el tema que ha escogido. En cuanto a los referentes artísticos reivindicamos la importancia de incorporar artistas contemporáneos, cuyos lenguajes y técnicas, según María Acaso (2015, p. 163), encajan perfectamente con la estética y los imaginarios de nuestros estudiantes. Esta misma autora afirma que las artes contemporáneas están muy poco presentes en las aulas y deberíamos recuperarlas por su poder narrativo y metafórico.

El proceso de investigación se desarrolla en espiral, con avances y aparentes retrocesos que obligan a los grupos a replantear sus decisiones y redefinir el problema o incluso abandonarlo por otro distinto. A partir de este trabajo, elaboran un mapa conceptual en base a sus conocimientos previos y la información que van obteniendo sobre el objeto de estudio.

A partir del mapa conceptual se inicia la síntesis entre los referentes teóricos y artísticos seleccionados, que constituye la tercera fase del proceso, la integración interdisciplinar. De aquí surge la idea que se materializa en la performance y se expresa a través de la interrelación de los lenguajes musical, corporal y plástico. Para ello se pueden utilizar recursos tecnológicos como editores de sonido o imagen, proyectores y pantallas, pero el principal recurso expresivo debe ser el movimiento de su propio cuerpo en interacción con el entorno y el grupo.

Un instrumento muy eficaz en la fase de integración y búsqueda de recursos expresivos es el storyboard, una representación gráfica donde los estudiantes dibujan la secuencia de movimientos de su performance. El storyboard es el paso previo a los ensayos porque permite discutir sobre la potencialidad comunicativa de la secuencia de movimientos en el espacio, su efectividad en la transmisión del mensaje y la interrelación que se establecerá entre el movimiento, el sonido y la imagen.

Finalmente, después de una fase de pruebas y ensayos comentados con el profesorado, los alumnos presentan su performance al resto del grupo en la última sesión del curso. Se trata de una metáfora expresada a través de múltiples lenguajes, en una acción artística en vivo, que pretende despertar una reacción en el espectador. En la presentación final es muy importante la actitud de los compañeros que actúan como público. La atención, el respeto y la reacción del auditorio es imprescindible para que cada grupo descubra la potencialidad comunicativa de su performance. Al final de la sesión se establece un diálogo entre los diversos grupos que han actuado para compartir las impresiones que cada performance ha provocado y valorar sus fortalezas y debili- 
dades a modo de evaluación formativa de carácter oral.

La actividad concluye con la fase de análisis crítico del resultado. Éste se realiza en una reflexión escrita que cada grupo incluye en su diario, a modo de autoevaluación del proceso creativo y su resultado, teniendo en cuenta los criterios establecidos en las rúbricas de evaluación (tabla 1 y tabla 2) que se han utilizado a lo largo de todo el proceso. Estas rúbricas se presentan al inicio del proceso creativo y se da la oportunidad al alumnado de modificar los criterios de forma consensuada con el profesorado. En las tutorías que se realizan a lo largo de todo el proceso creativo, los grupos valoran sus progresos en función de los criterios acordados y recibiendo feedback al respecto por parte del profesorado.

\begin{tabular}{|c|c|c|}
\hline \multicolumn{3}{|c|}{ Valorad del 1 al 5, siendo: 1 = Nada, 2 = Poco, 3 = Algo, 4 = Bastante, y 5 = Mucho. } \\
\hline & \begin{tabular}{|c|} 
Criterio \\
\end{tabular} & Valoración \\
\hline Originalidad & $\begin{array}{l}\text { El resultado es una nueva manera de acercarse al concepto } \\
\text { que se pretende expresar artísticamente. A pesar de incluir } \\
\text { las aportaciones del profesorado, se evidencia la } \\
\text { autenticidad del grupo. }\end{array}$ & \\
\hline $\begin{array}{l}\text { Experimentación } \\
\text { y flexibilidad }\end{array}$ & $\begin{array}{l}\text { Existe total disposición a experimentar en su proyecto, } \\
\text { trabajar desde otras perspectivas, ser flexible y no } \\
\text { mantenerse en el "espacio seguro". }\end{array}$ & \\
\hline Coherencia & $\begin{array}{l}\text { Nada es gratuito. Cada elemento tiene su significado. } \\
\text { Existe total coherencia (se trata de un todo estructurado y } \\
\text { no de elementos aislados) y consistencia entre los recursos } \\
\text { expresivos y los fundamentos teóricos del proyecto. }\end{array}$ & \\
\hline $\begin{array}{l}\text { Valores estéticos y } \\
\text { riqueza simbólica }\end{array}$ & $\begin{array}{l}\text { Expresa el máximo con los mínimos elementos (menos, es } \\
\text { más). Usa de manera equilibrada (ni sobra ni falta nada), } \\
\text { original (creado expresamente o versionado a partir de la } \\
\text { obra de otros autores) y técnicamente correcta los } \\
\text { diferentes recursos expresivos (audiovisuales y corporales). } \\
\text { El producto contiene información interna y códigos que el } \\
\text { público necesita decodificar (lenguaje metafórico). }\end{array}$ & \\
\hline $\begin{array}{l}\text { Capacidad de } \\
\text { provocación }\end{array}$ & $\begin{array}{l}\text { El resultado permite ampliar la reflexión sobre el tema } \\
\text { propuesto, abre nuevas maneras de enfocarlo, transmite } \\
\text { emociones y aporta nuevos valores estéticos. }\end{array}$ & \\
\hline $\begin{array}{l}\text { Investigación y } \\
\text { trabajo autónomo }\end{array}$ & \begin{tabular}{|l|} 
El proyecto demuestra un exhaustivo conocimiento de \\
referentes teóricos y artísticos y establece claras conexiones \\
entre éstos y las aportaciones del grupo. Se evidencia un \\
notable trabajo autónomo de investigación.
\end{tabular} & \\
\hline Trabajo en equipo & $\begin{array}{l}\text { Se evidencia un fuerte trabajo en equipo a lo largo del } \\
\text { proceso, así como el desarrollo de habilidades } \\
\text { comunicativas grupales. Se participa con responsabilidad } \\
\text { grupal y equitativamente en el proyecto. Existe absoluta } \\
\text { colaboración para potenciar los resultados. }\end{array}$ & \\
\hline \multicolumn{3}{|l|}{ Justificación: } \\
\hline \multicolumn{3}{|c|}{ Fuente: Adaptada de Del Pozo (2012) } \\
\hline \multicolumn{3}{|c|}{$\begin{array}{l}\text { Tabla } 2 . \\
\text { Instrumento de evaluación de la expresión corporal. }\end{array}$} \\
\hline \multicolumn{3}{|c|}{$\begin{array}{l}\text { Valora del } 1 \text { al } 5 \text {, siendo } 1 \text { = Carencias importantes, } 2 \text { = Pequeñas carencias, } 3=\text { Suficiente, } 4 \\
\text { = Bueno, y } 5 \text { = Muy bien. }\end{array}$} \\
\hline & Criterio & Valoración \\
\hline $\begin{array}{l}\text { Ocupación del } \\
\text { espacio }\end{array}$ & $\begin{array}{l}\text { El uso del espacio (distribución, recorridos...) contribuye de } \\
\text { forma eficaz a la expresión de la idea que se pretende } \\
\text { comunicar. }\end{array}$ & \\
\hline $\begin{array}{l}\text { Coordinación } \\
\text { entre los } \\
\text { miembros del } \\
\text { grupo }\end{array}$ & $\begin{array}{l}\text { Existe una coordinación total entre todos los miembros del } \\
\text { grupo a lo largo de la propuesta. }\end{array}$ & \\
\hline $\begin{array}{l}\text { Expresividad y } \\
\text { comunicación }\end{array}$ & $\begin{array}{l}\text { Se realizan movimientos cualitativos y estéticos en la } \\
\text { representación de ideas, sensaciones y sentimientos que } \\
\text { captan la atención del espectador y consiguen comunicar el } \\
\text { mensaje que se pretende transmitir. }\end{array}$ & \\
\hline \begin{tabular}{l|l} 
Apoyo musical a \\
las intensidades \\
del movimiento
\end{tabular} & $\begin{array}{l}\text { Existe coherencia y coordinación entre la acción motora y el } \\
\text { apoyo musical o sonoro de la representación. }\end{array}$ & \\
\hline
\end{tabular}

Respecto a la calificación final de la performance, optamos por un sistema coherente con el aprendizaje cooperativo. En primer lugar, se acuerda con el alumnado el porcentaje de cada ítem de la rúbrica (tabla 1) en la nota final (calculada sobre 10). Cuando se ha obtenido la nota de cada performance, ésta se multiplica por el número de miembros de cada grupo y se aplica un sistema de calificación consensuada (Johnson \& Johnson, 2014). Este sistema consiste en dele- gar en el grupo la responsabilidad de repartir la puntuación entre sus componentes en función de su aportación al trabajo. De esta forma, cada estudiante obtiene una calificación según su participación, pero consensuada en el grupo.

\section{Conclusiones}

Tras varios años de codocencia en el módulo de Educación a través de la plástica, la música y la educación física: proyecto interdisciplinar, la experiencia compartida por el equipo docente nos lleva a afirmar que este proyecto contribuye a la formación personal y profesional de los futuros maestros porque los pone en situación de experimentar sus propias capacidades expresivas, con el doble objetivo de comunicar vivencias, emociones o ideas y de establecer relaciones constructivas en el grupo. Además, los capacita para poder liderar procesos similares en su futuro como docentes en la escuela.

Uno de los mayores retos con los que se enfrentan los estudiantes a lo largo del proceso creativo que se ha llevado a cabo, está relacionado con la dificultad de integrar contenidos y lenguajes propios de distintas disciplinas para crear una metáfora. En este sentido, los referentes artísticos aportados por el equipo docente son imprescindibles como ejemplos de síntesis interdisciplinar expresada a través de los lenguajes corporal, musical y visual y plástico.

En esta experiencia, la codocencia es una condición indispensable para garantizar la perspectiva interdisciplinar en todo el proceso y, además, ofrece a los estudiantes la posibilidad de escuchar las sugerencias de distintos profesores que no necesariamente están de acuerdo en todas sus orientaciones. Esta diversidad de opiniones en el equipo docente puede desconcertar a los estudiantes al principio, pero consideramos que el diálogo respetuoso entre profesionales de distintas disciplinas enriquece el proyecto, obliga a los estudiantes a tomar y justificar sus propias decisiones $\mathrm{y}$, a su vez, constituye un ejemplo de colaboración docente para los futuros maestros.

La codocencia requiere la coordinación del profesorado en el diseño, implementación y evaluación del proyecto artístico interdisciplinar. Ello implica disponer de tiempo compartido, no únicamente durante las sesiones de clase, sino también durante la programación, preparación y continua revisión de nuestra práctica. Del mismo modo, el desarrollo de un proceso creativo a través de los lenguajes expresivos requiere de unas condiciones de espacio que permitan la práctica de los estudiantes más allá de los horarios de clase. En este sentido, es imprescindible que la institución educativa que apueste por los proyectos artísticos interdisciplinares y la codocencia, contemple en su organización la necesidad de amplias franjas horarias para la coordinación de los equipos docentes y de espacios adecuados para las reuniones y la práctica de los estudiantes.

A diferencia de otros trabajos en grupo que los estudiantes han realizado a lo largo de su formación académica, el desarrollo de la performance requiere la presencia, implicación y cooperación permanente de todos los componentes del grupo en cada fase del proceso. El proceso creativo reúne todas la condiciones del aprendizaje cooperativo: la interdependencia positiva, puesto que los estudiantes toman 
conciencia de que cada decisión los implica a todos y por ello debe ser discutida, consensuada y revisada conjuntamente; la interacción promotora, porque las sesiones en las que se avanza en el trabajo autónomo y las tutorías con el profesorado requieren la presencia física y una actitud constructiva de todos los componentes; la responsabilidad y valoración personal de cada componente que debe ser consciente de su contribución al trabajo grupal; el desarrollo de las habilidades interpersonales tales como la escucha activa, la flexibilidad y la resolución de conflictos, imprescindibles en una experiencia de aprendizaje nueva para los estudiantes que puede generar inseguridad y tensiones; y finalmente, el procesamiento grupal, de reflexión colectiva sobre el funcionamiento y eficacia del grupo, está implícito en la fase final de análisis crítico de los resultados y el procedimiento de evaluación formativa y consensuada.

La incorporación de recursos y instrumentos de evaluación formativa, integrados en este proceso de aprendizaje grupal y cooperativo, también se valora muy positivamente por parte de los propios estudiantes y docentes del módulo. En la evaluación final de la asignatura, como se recoge en Córdoba (2017), el alumnado ha expresado que el modelo de sesión basado en el trabajo autónomo de los grupos y las tutorías, ha contribuido positivamente en su proceso de aprendizaje porque les permite disponer de tiempo para avanzar en sus proyectos y recibir un feedback constante del profesorado. Por su parte, el equipo docente considera que la inclusión de procesos de autoevaluación periódica de los grupos a partir de criterios consensuados, genera una mayor implicación y autonomía de los grupos de trabajo, así como un análisis más profundo de cada una de las tareas.

El análisis detallado de la actividad que hemos presentado es el resultado de un proceso de práctica reflexiva compartido por el equipo de profesorado del módulo, que repercutirá en la mejora de la planificación, implementación y evaluación del proyecto interdisciplinar. Creemos que puede ser útil a aquellos profesionales de la formación inicial de maestros que incluyen o se plantean incluir en su práctica docente los proyectos artísticos interdisciplinares, el aprendizaje cooperativo y la evaluación formativa.

Entre las limitaciones de este estudio debemos destacar que se trata de la descripción y análisis de un caso único por lo que las conclusiones que se obtienen no se pueden generalizar ni extrapolar a otros contextos.

Como futuras líneas de investigación a partir de la propuesta que hemos presentado, nos planteamos, por un lado, futuros proyectos interdisciplinares que exploren los vínculos entre la educación física con otras áreas y, por otro, la posibilidad de fortalecer el sistema de evaluación y el aprendizaje cooperativo incluyendo dinámicas de coevaluación entre grupos de trabajo.

\section{Referencias}

Acaso, M. (2015) rEDUvolution. Hacer la revolución en la educación. Barcelona: Paidós.

Álvarez-Méndez, J. M. (2001). Evaluar para conocer, examinar para excluir. Madrid: Morata.

Batet, M. Ponti, F. \& Segarra, E. (2015). Crear i fer ampolles. Eines per educar la creativitat. Vilafranca del Penedés:
Andana

Bautista, A.; Tan, L.; Ponnusamy, L. \& Yau, X. (2015). Curriculum integration in arts education: connecting multiple art forms through the idea of 'space'. Journal of Curriculum Studies, 48(5), 610-629. doi: 10.1080/ 00220272.2015.1089940

Boix, V. (2016) Interdisciplinary learning: A cognitiveepistemological foundation. En R. Frodeman y J. Klein (Eds). Oxford Handbook of Interdisciplinarity. UK: Oxford University Press.

Cela, J. \& Domènech, J. (2016). Quines competències professionals ha de tenir un mestre avui? Revista Catalana de Pedagogia, 9, 62-72.

Civís, M. ( 2017). L’adequació del perfil dels mestres davant de necessitats de l'escola actual. Barcelona: Agència per a la Qualitat del Sistema Universitari de Catalunya. Universitat de Catalunya. Recuperado de http:// www.aqu.cat/doc/doc_50532612_1.pdf

Cook, L (2004). Co-Teaching: Principles, Practices, and Pragmatics. Albuquerque: New Mexico Public Education Department.

Córdoba, T. (2017). Una experiencia de evaluación formativa en el módulo de música, expresión plástica y corporal. Revista Infancia, Educación y Aprendizaje, 3(2), 544-549. doi:10.22370/ieya.2017.3.2.777

Córdoba, T.; López-Pastor, V. M. \& Sebastiani, E. M. (2018). ¿Por qué hago Evaluación Formativa en Educación Física? Relato autobiográfico de un docente. Estudios Pedagógicos, 44(2), 21-38. doi:10.4067/S071807052018000200021

Del Pozo, P. (2012). La rúbrica y los flashes en la evaluación de la expresión corporal. EmásF: revista digital de educación física, (17), 38-48.

EHEA (2018). Communiqué of the Conference of European Ministers Responsible for Higher Education, Paris, 2425 May 2018. Recuperado de http://www.ehea.info/ media.ehea.info/file/2018_Paris/7 7/1/ EHEAParis2018_Communique_final_952771.pdf

Fernández-Río, J. (2017). El Ciclo del Aprendizaje Cooperativo: una guía para implementar de manera efectiva el aprendizaje cooperativo en educación física. Retos. Nuevas tendencias en educación física, deporte y recreación, (32), 264-269. Recuperado de https://dialnet.unirioja.es/ servlet/articulo?codigo $=6352316$

Folch, C.; Capdevila, R. \& Prat, M. (2019). Percepción del Profesorado sobre una Experiencia Multidisciplinar: Arte y Ciencias en un Grado de Educación. Revista de Investigación y Docencia Universitaria. doi:10.19083/ ridu.2019.743

Follari, R. (2007). La interdisciplina en la docencia. En POLIS, Revista Latinoamericana, 6 (16), 1-12. Recuperado de https://www.redalyc.org/articulo.oa?id=30501603

Follari (2013). Acerca de la interdisciplina. Posibilidades y límites. INTERdisciplina, 1 (1). doi:10.22201/ ceiich.24485705e.2013.1.46517

Fontanet, A (2017). Adequació del perfil del mestre davant les necessitats socioeducatives actuals: consideracions i propostes per a la formació inicial. (Tesi doctoral. Universitat Ramon Llull, Catalunya). Recuperado de https://www.tdx.cat/handle/10803/404565 
Imbernón, F. \& Colén, M. T. (2015). Los vaivenes de la formación inicial del profesorado. Una reforma siempre inacabada. Tendencias Pedagógicas, 25, 57-76.

Johnson, D., \& Johnson, R. (2014). La evaluación en el aprendizaje cooperativo. Cómo mejorar la evaluación individual a través del grupo. Madrid: Ediciones SM.

Johnson, D.; Johnson, R. \& Holubec, E. (1999). Los nuevos círculos del aprendizaje. La cooperación en el aula y la escuela. Buenos Aires: Aique.

Kluth, P. \& Straut D. (2003). Do as we say and as we do. Teaching and modeling collaborative practice in the university classroom. Journal of Teacher Education, 54 (3), 228-240. doi:10.1177/0022487103054003005

López-Pastor, V. M. (Coord.) (2006). La Evaluación en Educación Física. Revisión de los modelos tradicionales y planteamiento de una alternativa: la evaluación formativa y compartida. Buenos Aires: Miño y Dávila.

López-Pastor, V. M. (Coord.) (2009). La Evaluación Formativa y Compartida en Docencia Universitaria: propuestas, técnicas, instrumentos y experiencias. Madrid: Narcea Ediciones. doi:10.4995/redu.2011.6185

López-Pastor, V. M.; Pérez-Brunicardi, D.; Manrique, J. C. \& Monjas, R. (2016). Los retos de la educación física en el siglo XXI. Retos, Nuevas tendencias en Educación Física, Deporte y Recreación, 29, 182-187.

López-Pastor, V. M., \& Pérez-Pueyo, A. (Coords.) (2017). Evaluación formativa y compartida en educación: experiencias de éxito en todas las etapas educativas. León: Universidad de León. Recuperado de http:// buleria.unileon.es/handle/10612/5999

MacBeath, J. (2013). Col·laborar, innovar i liderar. El futur de la professió docent. Barcelona: Fundació Jaume Bofill.

Marina, J.A., Pellicer, C. \& Manso, J. (2015). Libro blanco de la profesión docente y su entorno escolar. Recuperado de http://www.mecd.gob.es/mecd/dms/mecd/destaca- dos/libroblanco/libro-blanco-profesion-docente.pdf

Martínez, M. \& Prats, E. (Coords.) (2018). Millora per a la formació inicial per a la docència. Accions Estratègiques. Programa de Millora i Innovació en la Formació Inicial de Mestres, 4 (1). Recuperado de https://mif.cat/wpcontent/uploads/2017/01/AccionsEstratCAT.pdf

Pujolàs, P. (2008). El aprendizaje cooperativo. 9 ideas clave. Barcelona: Graó.

Santos-Guerra, M. A. (2015). La evaluación como aprendizaje. Madrid: Narcea.

Schleicher, A. (2015). Schools for 21st-Century Learners: Strong Leaders, Confident Teachers, Innovative Approaches. International Summit on the teaching profession. OECD publishing. doi:10.1787/9789264231191en

Suárez-Díaz, G. (2016). Co-enseñanza: concepciones y prácticas en profesores de unaFacultad de Educación en Perú. Revista Electrónica de Investigación Educativa, 18(1), 166-182. Recuperado de http://redie.uabc.mx/redie/article/ view/786

UNESCO (2015). Replantear la educación. ¿Hacia un bien común universal? París: UNESCO. doi:10.16925/ greylit.2051

Velázquez-Callado, C. (2010). Aprendizaje cooperativo en Educación Física. Fundamentos y aplicaciones prácticas. Barcelona: INDE.

Velázquez-Callado, C. (2015). Aprendizaje cooperativo en Educación Física: estado de la cuestión y propuesta de intervención. Retos: nuevas tendencias en educación física,deporte y recreación, (28), 234-239. doi:10.35376/ $10324 / 2823$

Velázquez, R. \& Hernández, J.L. (2005). La educación física y su imagen social a la luz de sus prácticas de evaluación y calificación del aprendizaje. Tándem: Didáctica de la Educación Física, 17, 7-20. Barcelona: Graó.

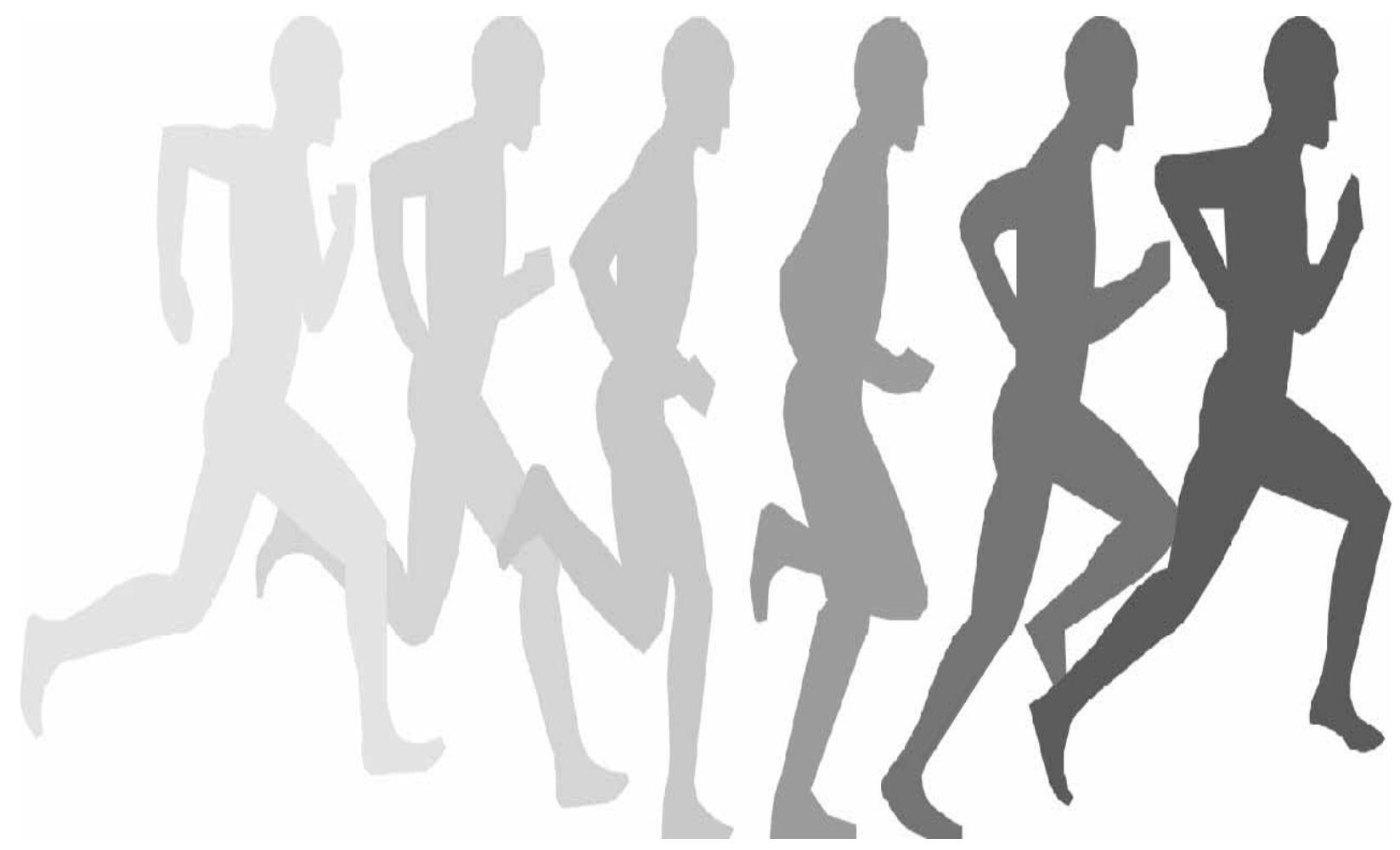

